\title{
The assessment of local lattice strains in alloys using total scattering
}

\author{
L.R. Owen ${ }^{\mathrm{a}, \mathrm{b}, *}$, H.J. Stone ${ }^{\mathrm{a}}$, H.Y. Playford ${ }^{\mathrm{b}}$ \\ ${ }^{a}$ Department of Materials Science and Metallurgy, University of Cambridge, CB3 OFS, UK \\ ${ }^{b}$ ISIS Facility, STFC Rutherford Appleton Laboratory, Didcot, Oxfordshire, OX11 OQX, UK
}

\begin{abstract}
The highly-strained lattice hypothesis in high-entropy alloys (HEAs) has led to an interest in local distortions created in substitutional solid-solution alloys. In this work, the use of total scattering for the assessment and analysis of local lattice strains in alloys is considered. Using two theoretical models, the variation in the width of peaks in the pair distribution function (PDF) with changes in composition, ordering and atomic radius is presented. Key practical considerations for the successful analysis of local lattice strains using this technique are discussed, with particular reference to sample preparation, instrumental and data processing effects. Further, the mitigation of errors in local-strain measurements caused by differences in the scattering length of constituent atoms is presented. This is concluded with a proposed methodology for the analysis of local strains using this technique.
\end{abstract}

Keywords: *Total scattering, Diffraction, Pair correlation function, Lattice strains

\section{Introduction}

It is commonly assumed that the substitution of atoms with others of different atomic radii leads to a local distortion field around the substituent atom. This strain field interacts with a moving dislocation, hindering its motion and

\footnotetext{
* Corresponding author

Email address: 1o250@cam.ac.uk (L.R. Owen)
} 
resulting in an increase in the strength of the material. Recently, the extensive interest in the novel metallurgical field of high-entropy alloys has extended this argument. It has been suggested that the mixing of multiple atomic species of differing atomic radii results in a 'highly-distorted lattice' [1, 2, 3]. The high level of local offsite displacement, or strain, exhibited by such a lattice is often cited as the explanation for the increased strength of many of this family of materials compared with compositionally less complex alloys. However, direct observations of such local strains in materials are relatively sparse in the literature.

In our previous work, a case study [4] was presented in which the total scattering technique, was used to assess the local lattice strain in the characteristic HEA CrMnFeCoNi (Cantor's alloy [5]). The study demonstrated the potential for using pair distribution functions (PDF) obtained from total scattering measurements to provide insight into the level of local strains exhibited in a lattice. The measurements made suggested that the lattice of Cantor's alloy [5] does not contain a comparatively higher level of strain than other compositionally simpler materials. If this result holds true for other HEAs, then their strengthening cannot be attributed to anomalous levels of strain and alternative mechanisms are required to explain the observed effects.

A full understanding of lattice distortions and their effects on the structure is key to understanding the structure-property relationship of alloys. As a probe of the local-environment, total scattering analyses allow the direct observation of such local effects and have already been used to provide new understanding and insight into a host of interesting complex materials systems (see e.g. [6]). However, for the technique to be used systematically for the study of local distortions in alloys a robust methodology is required. This paper seeks to provide such a methodology by answering two questions. Firstly, is the PDF susceptible to changes in the local level of strain exhibited in a lattice due to variation of concentration and radii of substituting species? Second, what practical steps need to be considered to ensure an accurate determination of local lattice strains? 


\section{Background theory}

It is commonly understood that dynamic thermal displacements away from idealised atomic positions in a structure results in a decay in the intensity of the Bragg peaks at high- $Q$ ( $Q$ is the magnitude of the scattering vector $\bar{Q}, Q=\frac{2 \pi}{d}$, where $d$ is the interplanar spacing in the lattice). This effect can be successfully described mathematically by a decaying form factor using a Debye-Waller factor [7, 8]. However, it should be noted that the total scattering intensity is not lost, but rather redistributed into diffuse scattering features that appear at the base of the Bragg peaks.

The effect of static distortions on a powder diffraction pattern would be expected to be analogous to a thermal Debye-Waller effect; manifesting as a decrease in the intensity of the Bragg peaks with an accompanying increase in the observed diffuse scattering. Unlike the standard micro-strains discussed in crystallographic literature, these local strains produced by the atomic substitution would not, therefore, be expected to produce broadening of the Bragg peaks, but rather a damping in the intensity of peaks at high- $Q$. Practically, the combined effects of static and dynamic displacements will therefore be subsumed into the $U_{\text {iso }}$ (isotropic Debye-Waller factor parameter) term of a Rietveld refinement. However, a direct measure of the local distortions in the system should still be possible from analysis of the diffuse scattering.

The total scattering technique, which is an extension of traditional powder methods, involves a simultaneous consideration of both the Bragg and diffuse components of scattering.

For neutrons, the total scattering function of a material, $F(Q)$, is given by:

$$
F(Q)=\rho_{0} \int_{0}^{\infty} 4 \pi r^{2} G(r) \frac{\sin Q r}{Q r} d r
$$

in which $Q$ is the magnitude of the scattering vector and $r$ is the instantaneous atomic position, $\rho_{0}$ is the average density of the structure and:

$$
G(r)=\sum_{i, j} c_{i} c_{j} \bar{b}_{i} \bar{b}_{j}\left(g_{i j}(r)-1\right)
$$


where $i$ and $j$ are atomic labels, $c_{i}$ is the concentration of species $i, \overline{b_{i}}$ the neutron scattering length of species $i$ and the $g_{i j}(r)$ terms are the partial pair distribution functions. The partial pair distribution functions are related to the spatial distribution of atoms by the expression:

$$
g_{i j}(r)=\frac{n_{i j}(r)}{4 \pi r^{2} \rho_{i} d r}
$$

where $n_{i j}(r)$ is the number of atoms lying within $r$ and $r+d r$ from an atom $i$, and $\rho_{i}=c_{i} \rho_{0}$, where $\rho_{0}$ is the atomic number density and $c_{i}$ the concentration of species $i$.

Consequently, the function $G(r)$, often referred to as the pair distribution function $(\mathrm{PDF})$, is a weighted histogram of the interatomic distances in a material. Practically, the PDF may be obtained by the inverse Fourier transform of Eq. 2, given by:

$$
G(r)=\frac{1}{(2 \pi)^{3} \rho_{0}} \int_{0}^{\infty} 4 \pi Q^{2} F(Q) \frac{\sin Q r}{Q r} d Q
$$

A peak in the pair distribution function will indicate atomic species separated by that distance. If the peak is assumed to be Gaussian (which is often practically found to be the case), the maximum of the peak will yield the average interatomic distance, whilst the peak area will be a function of the number and type of correlations at that distance. Importantly, the peak width will be a function of the distribution in the interatomic distances around the average interatomic distance (given by the peak maximum).

In a substitutional solid-solution, the distortion field created by the introduction of a solute atom with a different atomic radius, will lead to a shift of the atoms from their idealised average atomic positions - referred to as local lattice strain. Such displacements would normally be described by an atomic displacement parameter, $U$, which is the mean square displacement of the atoms in the system away from their ideal locations. This change in the magnitude of the off-site displacements will change the distribution of the interatomic distances, and thus the width of peaks in the PDF (see Fig. 1)

The measurement of the change in peak widths is different to the majority 

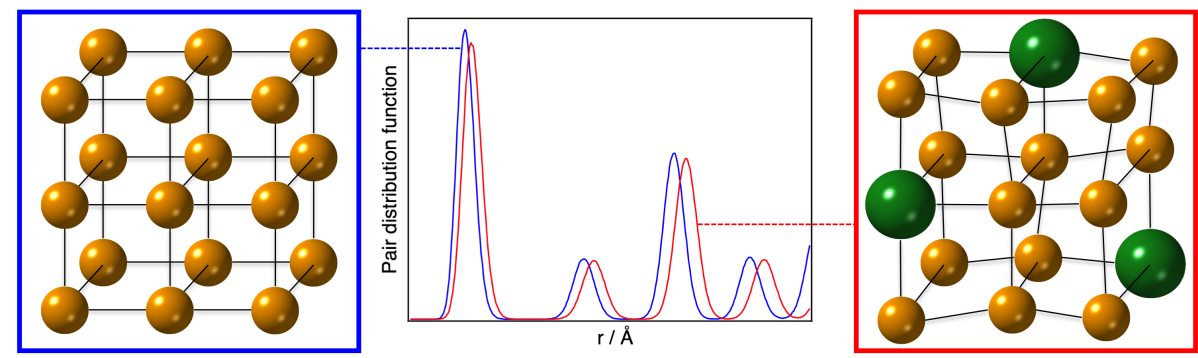

Figure 1: Demonstrative illustration of the change in the PDF on the substitution of atoms with a different atomic radius. The increase in local lattice strain results in an increase in peak width. The lattice also expands to accommodate the larger atoms, resulting in a shift in the peak positions. Colour version available online.

of studies carried out using PDFs, which normally follow larger changes such as the shift in peak positions or the appearance of new features in the PDF. It is further distinct from the previous studies of short-range order (SRO) in alloys [9, 10] where variations in peak areas were recorded, as a result of the change in occupation of different coordination shells.

\section{Changes in the PDF due to lattice distortion}

\subsection{The relationship between lattice strain and PDF peak width}

It is commonly assumed that the displacements of atoms away from their ideal lattice positions, $u$, can be modelled by a Gaussian distribution of the form:

$$
f(u)=N_{u} \exp -\left(\frac{u^{2}}{2 \sigma_{u}^{2}}\right)
$$

where $N_{u}$ is a scaling factor, and $\sigma_{u}$ is the width of the distribution and hence the numerical measure of the displacements in the material.

Provided that all atoms obey this distribution, their interatomic distance, $r$, will be described by the convolution of Eq. 5 with a similar function centred at the average interatomic distance of a given shell, $\mu_{r}$. The corresponding peak in the PDF will therefore be given by:

$$
f(r)=N_{r} \exp -\left[\frac{\left(r-\mu_{r}\right)^{2}}{2 \sigma_{r}^{2}}\right]
$$


where

$$
\sigma_{r}=\sqrt{2} \sigma_{u}
$$

and

$$
N_{r}=\sqrt{\pi} N_{u}^{2} \sigma_{u}
$$

As the PDF peak width is a scalar multiple of the width of the distribution of the atomic displacements, the PDF peak width can be used as a quantitative indicator of the offsite displacement of the atoms. Therefore, throughout this paper the PDF peak width will be used as a proxy for the offsite displacement.

It should be noted that the offsite displacements exhibited by the atoms will be a combination of local lattice strains and thermal components. The magnitude of the offsite displacements from static effects, $\sigma_{S}$, and thermal displacements, $\sigma_{D}$, may be related to the overall offsite displacement, $\sigma_{u}$, by:

$$
\sigma_{u}^{2}=\sigma_{S}^{2}+\sigma_{D}^{2}
$$

In the examples considered in the following sections, the homologous temperature of the samples was taken as being constant, and hence the magnitude of the thermal displacements would be invariant. Therefore, changes in the PDF peak width should be attributable to changes in local lattice strain (see Fig. 11. Based on the definition of these types of local strain, it is common to quote the magnitude of $\sigma_{S}$ as a quantitative measure of the displacement exhibited by atoms in the structure due to local static effects. This can be converted to a dimensionless quantity, more consistent with definitions of macroscopic engineering strains, by taking the ratio to the average atomic radius of the material:

$$
\varepsilon_{\mathrm{L}}=\frac{\sigma_{S}}{r_{\mathrm{Av}}}
$$

The average atomic radius, $r_{\mathrm{Av}}$, will be most commonly calculated from the experimentally measured lattice parameter and assuming a hard sphere model. 


\subsection{Vegard's relation}

If an atom with a larger atomic radius is substituted into a monatomic structure, the lattice expands to accommodate the larger atomic species (the converse is true for the substitution of atoms with smaller atomic radii). This can be observed in the change in the lattice parameter extracted from fitting of the Bragg pattern or traditional powder indexing methods. For binary alloys, the variation of lattice parameter with concentration of the substituting species is often approximately linear, and this trend is known as Vegard's relation [11]. However, it should be noted that whilst Vegard's relation often predicts the lattice parameters of binary alloys fairly well, it does not necessarily hold for higher order systems. Nevertheless, in the absence of a more accurate model, the lattice parameter of a higher order system is often still predicted using a weighted average of the atomic radii of the individual components.

For the theoretical binary alloys considered in this work it will be assumed that Vegard's relation holds for the average structure, i.e. the unit cell of the material. Consequently, it is expected that the positions of the peak maxima in the PDF will shift when substituting with an atom of a different atomic radius (see Fig. 1). In this paper, the predictions will be limited to a consideration of $f c c$ structures only, but a similar treatment could equally be applied to any crystal structure.

\subsection{Variation in the distribution of bond lengths}

As noted previously, the local lattice strain is a measure of the distribution of the average offsite displacement of atoms, and hence the nearest neighbour interatomic distances. It can be imagined that the substitution of atoms (replacing $\mathrm{A}$ atoms with atoms of $\mathrm{B}$ ), with a differing atomic radius, into a lattice could have two main effects on the distribution of interatomic distances. Firstly, there may be a change in the average interatomic distances of the different pairs of atoms, i.e. the $\mathrm{AB}$ and $\mathrm{BB}$ average distances may be different from the $\mathrm{AA}$ bond length. Secondly, the width of the distribution of the interatomic distances may change, compared with the width of the pure monatomic sample. 
In reality, the observed effect is likely to be a combination of these two effects. To demonstrate the changes that might be expected on substitution, two simple models can be proposed:

- Sum of Gaussians Model (SGM) - The distributions of the AA, AB and BB pair distances are assumed to be independent Gaussian distributions that can be summed, Eq. 2, to yield a peak in the PDF. This model assumes that no relaxation occurs in the system and is only capable of calculating the first peak in a PDF. To account for higher order correlations, the spatial relationship and radii of the correlating atom types, and the atoms located at intermediate positions in the lattice would need to be considered - the calculation of which rapidly becomes complex. Whilst these are obviously limitations, the model is useful in providing an upper bound on the maximum effect on the PDF that might be observed upon substitution.

- Spring model (SM) - The system is modelled as a collection of hard spheres connected by springs, with energies described by a Morse potential. By energetically relaxing the system, a more physically realistic representation of what is likely to occur on substitution of B into A may be obtained. Importantly, this enables the calculation of the PDF of the structure up to a desired point in $r$, and not the first peak in isolation.

Full details of the two models and how the calculations were carried out can be found in the supplementary information. In formulating these models it was assumed that all of the alloys demonstrate the same magnitude of thermal oscillations, reflecting real materials measured at the same homologous temperature. Using these two models it is possible to demonstrate how the PDF peaks may vary as a result of changes in alloy composition (e.g. relative concentrations, atomic radii etc.).

To obtain meaningful information about the local lattice strain, it is necessary that quantitative characterisation of the PDF peaks is performed. One potential method involves fitting the calculated PDF peaks with a Gaussian 
function of the form 11 unlikely to be strictly Gaussian in nature they can still be reasonably well fitted with this function, and the relevant parameters obtained with a corresponding error. Alternatively, the full width at half maximum (FWHM) of the peak can be measured. The latter approach is a more general method as it makes no assumptions about the shape of the peak. The two methods can be compared using the relationship FWHM $=2 \sigma \sqrt{2 \ln 2}$, where $\sigma$ is the Gaussian width parameter. As the two methods are found practically to produce little difference in the measured values, the former method will be employed here, as the fitting algorithms used provide an estimate in the error of the fitted distribution.

\subsection{Variation with concentration}

To establish the effect of varying concentration, a series of theoretical $f c c$ alloys of composition $\mathrm{A}_{1-x} \mathrm{~B}_{x}$, where $x=0,0.05,0.10 \ldots 1.00$ were considered. The radii of the $\mathrm{A}$ and $\mathrm{B}$ atoms were taken to be $r_{\mathrm{A}}=1.245 \AA$ (approximately that of nickel) and $r_{\mathrm{b}}=1.30725 \AA$ i.e. the radius of $\mathrm{B}$ is $5 \%$ larger than that of A (a typical radius difference, well within the bounds of stability of solid solution suggested by Hume-Rothery [12]). The lattice parameters of the A-B alloys were calculated using Vegard's relation and the nearest neighbour bond distances were then determined by a hard sphere model as $r_{\mathrm{AA}}=2.49000 \AA$, $r_{\mathrm{AB}}=2.55225 \AA$ and $r_{\mathrm{BB}}=2.61450 \AA$.

The two proposed peak calculation models were applied and the variation in predicted PDF peak widths with concentration were obtained using the Gaussian function fits. The modelling was carried out for three cases to demonstrate the variations caused by differences in the scattering lengths of the atoms. The three separate cases considered are: a) $b_{\mathrm{A}}=b_{\mathrm{B}}$, b) $2 b_{\mathrm{A}}=b_{\mathrm{B}}$ (i.e. $b_{\mathrm{A}}<b_{\mathrm{B}}$ )

\footnotetext{
${ }^{1}$ It is worth noting that this is the non-normalised version of this function where $N$ is a scaling factor. This form of the Gaussian has been used in lieu of the more usual function (which contains $a, b$ and $c$ parameters), to prevent confusion between symbols (e.g. the scattering length $b_{i}$ )
} 
and c) $b_{\mathrm{A}}=2 b_{\mathrm{B}}$ (i.e. $b_{\mathrm{A}}>b_{\mathrm{B}}$ ). The resultant PDF peak parameters and calculated local lattice strain from the SGM (fitting with a Gaussian) are shown in Fig. 2(a), (b) and (c). Considering the case where $b_{\mathrm{A}}=b_{\mathrm{B}}$ (black) there is an observable increase in the peak width and local strain as the concentration of $\mathrm{B}$ increases, up to a maximum at the equiatomic concentration, as would be expected. Likewise, the peak maximum shows the linear increase predicted by Vegard's relation.
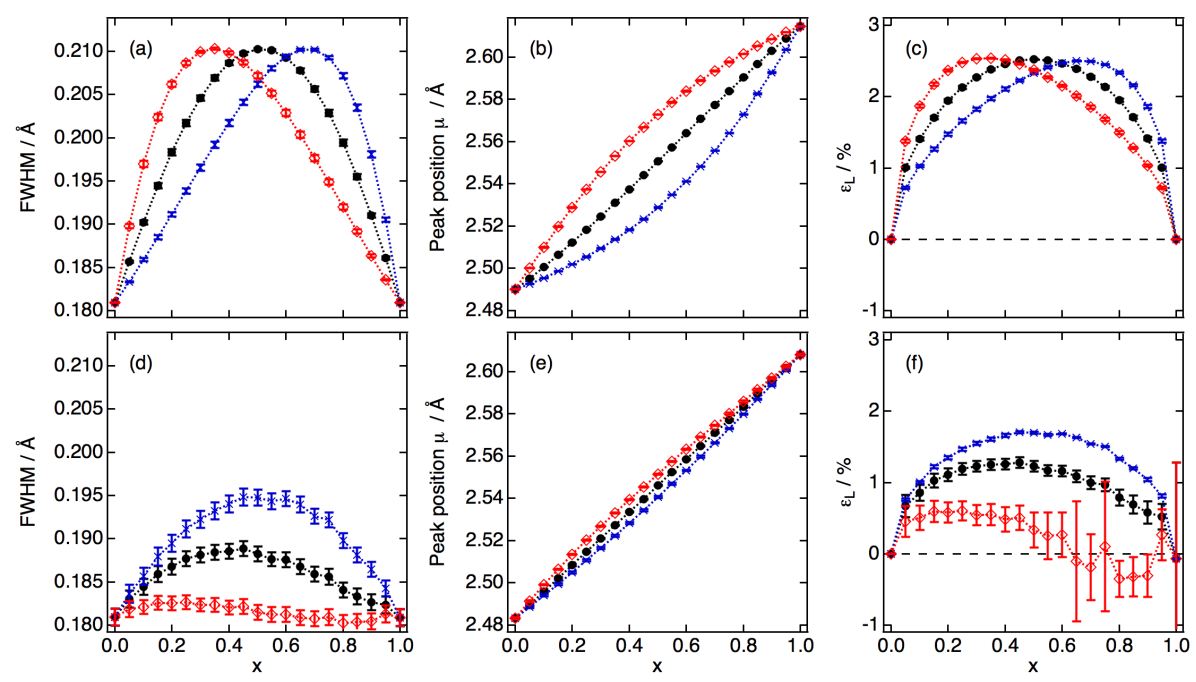

Figure 2: Variation in peak parameters for the first peak in the PDF with varying concentration for the two models, (a), (b) and (c) predicted from SGM, (d), (e) and (f) from SM, fitting the resultant peak with a Gaussian. (a) and (d) show the calculated full width at halfmaximum; (b) and (e) the peak position or $\mu$ parameter; (c) and (f) show the calculated local lattice strain from eqs. 9 and 10 Black line (circles) indicates $b_{\mathrm{A}}=b_{\mathrm{B}}$, red line (diamonds) $2 b_{\mathrm{A}}=b_{\mathrm{B}}$ and blue line (crosses) $b_{\mathrm{A}}=2 b_{\mathrm{B}}$. Colour version available online.

However, considering the cases where $b_{\mathrm{A}} \neq b_{\mathrm{B}}$ it is apparent that there is a skew to the distribution of PDF peak width with A-B alloy composition. From this, it can be seen that the measured peak width will vary as the relative magnitudes of the scattering lengths of the components changes. This may result in an under or over estimate of the local strain in the lattice, depending on the relative magnitude of the component scattering lengths, as is seen in 
panel (c) (in this example, up to $\sim 40 \%$ error in the observed strain). This is problematic, as it means that the peak width cannot be directly related to the local lattice strain, without a consideration of the relative scattering lengths. Methods for dealing with this issue are discussed in Section 4.1.

The result from the SM predictions are shown in Fig. 2(d), (e) and (f). Comparing Fig. 2(b) and (e) it can be seen that both models predict a similar variation in the peak maximum, although a much smaller change is apparent in the SM owing to the relaxation of the system. However, a much greater variation is observed between the peak widths predicted by the two models (Fig. 2(a) and $(d))$. Most apparent is the overall reduction in the maximum strain shown (compared with the SGM), Fig. 2(f), which is again the result of relaxation in the system. In addition, differences in the relative scattering lengths now not only change the skew of the distributions, but also the maximum change in the FWHM as the concentration varies. From Fig. 2(d) it seems that if $b_{\mathrm{A}}<b_{\mathrm{B}}$ the peak width change is minimal, whereas if $b_{\mathrm{A}}>b_{\mathrm{B}}$ there is a large change in the peak width.

This variation in peak width suggests that for the SM, the peak width is being dominated by the variation in the AA partial - i.e. the partial with the closest interatomic distance. This is most likely the result of the asymmetric nature of the Morse potentials used. As the system relaxes, the AA distribution will shift to higher- $r$ values, whilst the BB distribution will reduce to lower- $r$ values. The asymmetry of the Morse potential will mean it is energetically more favourable to expand the AA partial to high $r$ than to expand the BB partial to lower $r$. Consequently the AA partial will broaden more and so will have a greater effect on the overall peak width. Hence, varying the relative scattering lengths of the partials will change the maximum observed in the peak width.

Considering the local lattice strains, there is comparatively little variation in the observed local strain with concentration in the $b_{\mathrm{A}}<b_{\mathrm{B}}$ case, and an increase in the observed local strain in the $b_{\mathrm{A}}>b_{\mathrm{B}}$ case, as a result of the changes in peak width. Indeed in the former case, the observed local lattice strain appears to become 'negative'. A note should be made that a negative 
local lattice strain is obviously neither physically nor mathematically possible. It indicates that the observed peak width is smaller than that caused by thermal oscillations in the system alone, which would mean that $\sigma_{S}^{2}<0$ (see eq. 9 ). The negative local strain plotted is calculated as $-\sqrt{\left|\sigma_{S}^{2}\right|}$ and demonstrates that there is an unaccounted for effect on the data (in this case the scattering lengths). Importantly, this further highlights the necessity for corrections to minimise the effect of scattering length on the observed peak widths of the system, see Section 4.1. Finally, it should be noted that the error on the local strain increases as it approaches zero. This is a consequence of the calculation of the local strain. The error in the calculated peak width is effectively constant, but as the local strain is a function of the difference between the peak width and the broadening caused by thermal displacements, in the limit as the local strain approaches zero the error will increase to infinity.

\subsection{Constant concentration, varying atomic radii}

Thus far, the variation in peak width under different levels of substitution of an atom with a fixed atomic radius has been considered. However, it is also useful to consider the change that might be observed for a series of alloys with the same concentration, but where the radius of the substituting atom is varied. Using the two models suggested previously, a similar analysis can be carried out. In this case, the radius of the substituting atom was varied in ratio to the solute atom radius in steps of $1 \%$ from 0 to $15 \%$. The maximum value was chosen to reflect the maximum difference in atomic radius suggested by Hume-Rothery [12] for which complete solid solution could be expected in a binary alloy. The resultant variation in width is shown for the two models in Fig. 3

The top axis shows the value of the $\delta$-parameter for the different alloys. Whilst, for binary alloys it is possible to quote the percentage difference in atomic radii of the two components, for higher order systems this is not possible. The $\delta$-parameter, developed for multicomponent alloys such as HEAs, provides a measure of the variation in atomic radius for alloys of any complexity enabling the comparison of more complicated alloy systems to simple 

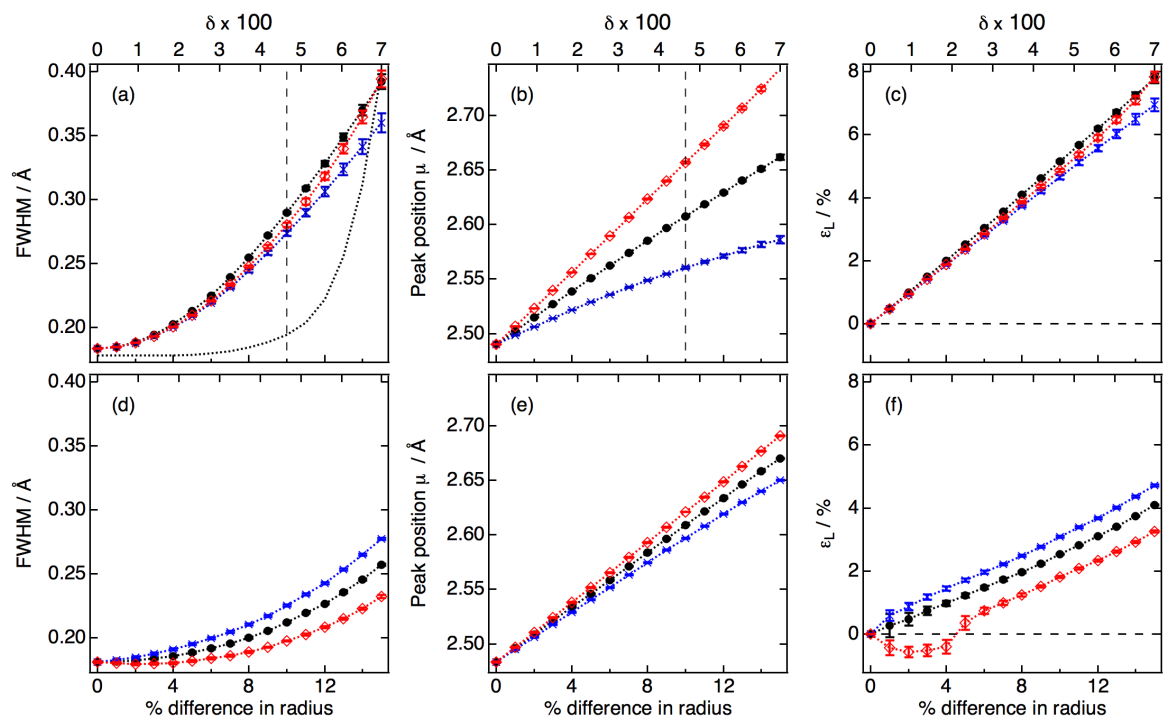

Figure 3: Variation in peak parameters for the first peak in the PDF with radius of the substituting atom for the two models for an alloy of fixed composition $\mathrm{AB}$, (a), (b) and (c) predicted using the SGM, (d), (e) and (f) from the SM, fitting the resultant peak with a Gaussian. (a) and (d) show the calculated full width at half-maximum; (b) and (e) the peak position or $\mu$ parameter; (c) and (f) show the calculated local lattice strain from eqs. 9 and 10 Dotted line on (a) demonstrates the R-squared of the fit for the $b_{\mathrm{A}}=b_{\mathrm{B}}$ SGM. Dashed line on graph indicates the region above which the SGM produces peaks that are no longer well described by fitting with a single Gaussian peak. Black line (circles) indicates $b_{\mathrm{A}}=b_{\mathrm{B}}$, red line (diamonds) $2 b_{\mathrm{A}}=b_{\mathrm{B}}$ and blue line (crosses) $b_{\mathrm{A}}=2 b_{\mathrm{B}}$. Colour version available online 
binaries. The parameter is defined as:

$$
\delta=\sqrt{\sum c_{i}\left(1-\frac{r_{i}}{r_{\mathrm{av}}}\right)^{2}}
$$

where:

$$
r_{\mathrm{av}}=\sum c_{i} r_{i}
$$

where $i$ is an atomic species label, $r_{i}$ and $c_{i}$ are the radius and concentration of atom species $i$ respectively.

Fig. 3(a) and (b) show the results of the predicted PDF peak widths and positions from the SGM. The dotted line indicates the values of $r$ (and $\delta$ ) for which the predicted first peak, from the SGM, is no longer well described by fitting with a single Gaussian. It should be noted that the errors displayed on the graph are errors in the fit, not the R-squared quality of the fit. The dotted line shows the R-squared for the equal weighted SGM prediction, from which the breakdown in quality of fit at high $\%$ differences in atomic radius for this model can be seen (i.e. when the peak is no longer well described by a single gaussian function). For the predictions made using the SM, there is no associated dotted line, due to the relaxation of the bonds in the system resulting in a distribution that remains well described by a Gaussian function for all $\delta$ values considered. Panels (a) and (d) show that for both models, and for all of the studied relative scattering length cases, there is the expected increase in the observed peak width with increasing difference in the size of the atomic radius and that it is well described by a quadratic expression. It can be seen that in all the cases, the Gaussian model predicts a much greater lattice strain than that predicted by the SM, panels (c) and (f). However, this can be easily explained by the lack of relaxation in the SGM. Again it is worth noting that in the SM without accounting for the scattering lengths a 'negative' local strain is predicted for the case where $b_{\mathrm{A}}<b_{\mathrm{B}}$.

The two different models also predict different FWHM values depending on the relative scattering lengths of the materials (with an error of up to $\sim 10 \%$ in this example). Whilst for the SGM, the equal scattering lengths case shows a 
maximum value; for the SM the peak width is under or over predicted depending on the relative sizes of $b_{\mathrm{A}}$ and $b_{\mathrm{B}}$. This is also reflected in the calculated local strains where, for a given difference in radius, the SGM demonstrates the maximum strain for the equal scattering length case, whilst for the SM this case yields an intermediate calculated strain. Note that these orders are consistent with those observed in Fig. 2 for the 50 at. \% cases. Without real data for comparison, it is difficult to say which of these is actually observed. However, it does again highlight the fact that some correction will be required for the relative magnitudes of the scattering lengths if the data is to be processed effectively to obtain quantitative information (See Section 4.1).

Fig. 3(b) and (e) show the variation in the observed peak maximum from the first PDF peak from the two models. Note that for both models the black line (the equally weighted scattering length cases) show the approximately linear increase that is expected for Vegard's relation. Changing the relative atomic scattering lengths results in a change to the observed peak maximum. For both models it suggests that if the scattering length of the largest atom is greater than that of the smaller atom, the peak maximum will occur at a distance greater than would be expected given the lattice parameter of the material. The converse is also true if the scattering length of the smallest atom is greater than that of the larger atom.

If the partials were weighted equally, then the smallest AA partial will have the same contribution to the peak as the largest BB partial. Consequently, when summed to yield the PDF peak, a symmetric peak will result. Increasing the relative weight of the highest- $r$ partial compared to the lowest partial will lead, when summed, to the shift in intensity to higher- $r$. The resultant peak will therefore be asymmetric with a shift in peak maximum to higher $r$ compared with the equal scattering length case.

The previous simulations were carried out for a 50:50 equiatomic binary. The same simulations and calculations can be carried out to demonstrate the expected variation with concentration (subject to equal scattering lengths). This can be seen in Fig. 4. For the SGM, shown in (a), (b) and (c), there is the 
expected increase in the peak width, local strain and peak position $\mu$ with increasing concentration of substituent. Note that for high values of $\delta$, Vegard's relation appears to break down, but this is only in the region that is no longer well described by the model (beyond the dashed line).
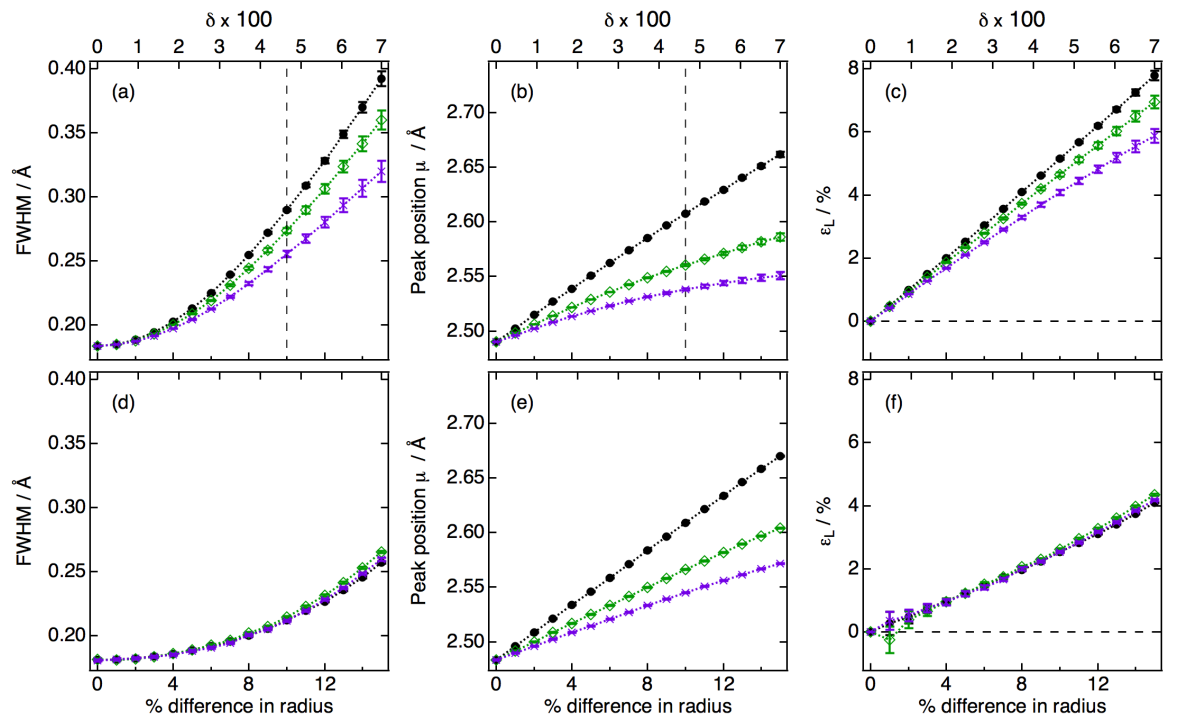

Figure 4: Variation in peak parameters for the first peak in the PDF with radius of the substituting atom using the two models, (a), (b) and (c) predicted from the SGM, (d), (e) and (f) from the SM, fitting the resultant peak with a Gaussian. (a) and (d) show the calculated full width at half-maximum; (b) and (e) the peak position or $\mu$ parameter; (c) and (f) show the calculated local lattice strain from eqs. 9 and 10 For all $b_{\mathrm{A}}=b_{\mathrm{B}}$. Dotted line on graph indicates the region above which the SGM produces peaks that are no longer well described by fitting with a single Gaussian peak. Black line (circles) indicates AB, green line (diamonds) $\mathrm{A}_{2} \mathrm{~B}$ and purple line (crosses) $\mathrm{A}_{3} \mathrm{~B}$. Colour version available online.

From the SM, panels (d), (e) and (f) it can be seen that the increase in peak width and local lattice strain is much less than that in the SGM case, but as stated previously this can be ascribed to the relaxation in this model. However, the change in the width and local lattice strain with composition is not as apparent. Comparing with Fig. 2(d) it can be seen that this is due to the relatively shallow change that occurs in the peak width as the concentration is varied from 20-50 at. \%B for the equal scattering length case. Again the peak 
maximum is well described by the linear nature of Vegards relation.

Overall it is important to note that the observed peak width and hence also the strain increases quadratically as the radius of the substituting atom increases. However, variations in the relative scattering lengths will result in slightly incorrect measured strain values obtained from the peak widths, and a slight correction will be required if accurate values are sought (See Section 4.1).

\subsection{Correlated motion}

The consideration of local strain within a lattice has, so far, been limited to the first coordination shell. However, it may be expected that a variation in the local strain measured from other peaks in the PDF will change when moving further out in $r$. For the same set of structures used to calculate Fig. 2 the variation in the peak width in the successive shells was obtained. Note that this was only possible using the large boxes generated from the SM. The SGM is restricted to a consideration of only the first shell effect, unless the effect of the intermediate atomic types and correlations can be made (as noted previously). The plot of the variation in peak widths with concentration for the first 5 peaks in the PDF is shown in Fig. 5(a). As can be seen from the graph, all the peaks exhibit a similar variation with elemental concentration. Importantly, however, there is a notable difference in the relative magnitudes of the peak widths, with the first shell showing a much narrower peak width than the subsequent shells. This can be further demonstrated in Fig. 5(b) where the change in observed peak width with $r$ for the simulated pure A alloy data is plotted.

This variation between peak widths from different coordination shells is attributable to the effect of correlated motion. If the system were completely non-interacting then the atoms would all be free to move independently and

there would be no $r$-dependance on peak width. Conversely, if the system were bonded with rigid rods, the movement of an atom would move all other atoms in the structure and a series of delta function peaks would result. For real systems, the nature of bonding is intermediate between these two extremes, and can be thought of as a system of atoms connected by springs. If an atom in 

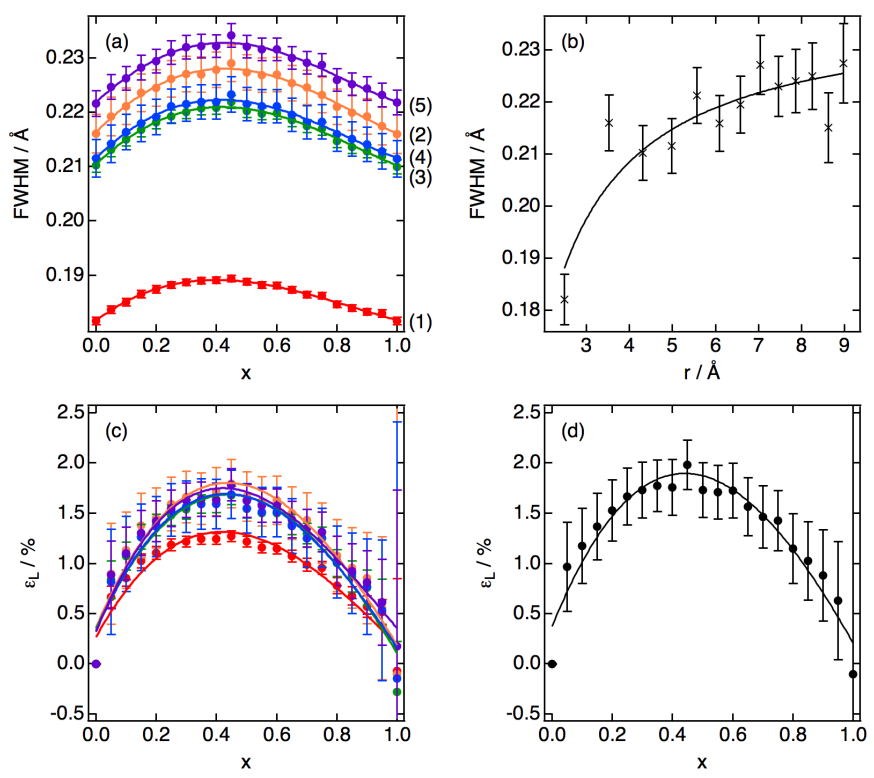

Figure 5: (a) Measured peak widths of the first 5 peaks in the PDF as a function of A-B alloy composition (labels on right hand side). (b) Variation in peak width in the PDF with $r$, simulated for a material containing only A atoms. Black line indicates fit with Eq. 13 (c) local lattice strain calculated from individual peaks, using eqs. 9 and 10 (d) local lattice strain calculated from $\sigma_{0}$ values from fitting Eq. 13 to the peak widths from the first 14 peaks in PDF generated using the SM.

the structure moves then the immediate neighbouring atoms will be displaced along with it, but some of the motion will be taken up by the extension of the spring or bond. For the subsequent neighbour shells there are more intermediate bonds between the moving atom and the atom of interest all of which will extend, leading to a lesser motion. Consequently the nearest neighbour PDF peaks will be narrower, and the correlations between atoms at greater distances will be broader.

The "small box" PDF analysis software PDFGui [13] uses the following expression to account for the variation in the overall peak width, $\sigma$, with $r$ that 
results from correlated motion.

$$
\sigma=\sigma_{0} \sqrt{1-\frac{\delta_{1}}{r}}
$$

where $\sigma_{0}$ the expected width of the peak as determined by the magnitude of the thermal oscillations in the system, $\delta_{1}$ is a parameter to account for correlated motion and $r$ the interatomic distance ( $\delta_{1} \sim 1.2$ for these materials). This function has been used in Fig. 5(b) to fit the observed data. The expression suggests that as $r \rightarrow \infty$ then $\sigma \rightarrow \sigma_{0}$. However, in the case of extreme local lattice distortion, such as is often proposed in high entropy alloy (HEA) systems, the magnitude of these distortions may be sufficiently large that the cumulative effect over successive shells results in a featureless PDF at high- $r$, i.e. the system tends towards an amorphous structure where only local coordination distances are maintained.

Fig. 5(c) and (d) show the calculated local lattice strain from this model. In (c) it can be seen that different values of strain are obtained depending on which shell is used. For lower order shells a lower local lattice strain is recorded. This is expected as for the lower order shells the correlated motion of atoms caused by the bonds will lead to smaller relative displacements and hence smaller measured strains. Fig. 5(d) shows the local lattice strain calculated from the $\sigma_{0}$ obtained from fitting Eq. 13 to the observed peak widths of the first fourteen peaks. This, therefore, acts as an upper bound on the measured strain in the system, as it effectivley models the displacements as if there was no correlated motion between the atoms. Which value of strain should be quoted, and used in subsequent property considerations, will depend on whether the correlated motion of atoms will need to be included and accounted for. It should be noted that the errors associated with fitting Eq. 13 are large and, as previously, as the strain tends to zero, the error tends to infinity - resulting in the large error shown with at $x=1.0$ (the error for $x=0$ is technically infinite - so is not plotted) 


\subsection{Variation with order}

It has been noted 10, 14 that ordering transitions in an alloy will result in a change in the observed area of a PDF peak. This is a consequence of the change in the numbers of the different atom types in a given coordination shell. However, assuming the locations of the occupied atomic sites are maintained in the transition and only order is changed, overall the total number of bonds at a given distance must be conserved. Therefore, if the number of A atoms in a coordination shell is reduced (with an accompanying reduction in the area of that peak in the partial), it must be compensated for by an increase in the occupation of another atomic species (and an increase in area in the peak of that partial). If the scattering lengths of all the atoms in the material are equal there should be no change in the observed total PDF under such an ordering. However, if the scattering lengths are different, this change in occupation will result in a change in the Faber-Ziman coefficients for the partials, and so an observed change in the area of the PDF.

A similar consideration can be made when considering the local lattice strain change on ordering. Assuming that a partial PDF peak can be modelled as a Gaussian, the area of such a peak will be:

$$
\text { Area }=\left(\sqrt{32 \pi^{3}}\right) \rho_{0} N \sigma\left[\sigma^{2}+\mu^{2}\right]
$$

where $N, \sigma$ and $\mu$ are Gaussian parameters, and $\rho$ is the density of the material. Assuming that the width, $\sigma$, and position, $\mu$, of the partial peaks remains constant under ordering then the $N$ parameter must change. If all of the partials in the system have the same $\mu$ and $\sigma$ parameters it follows that, irrespective of their weighting, the sum will be a scalar multiple (a linear combination) of the Gaussian functions that define the peaks in each of the individual partials. Consequently, it follows that there should be no change in the observed PDF peak width under ordering.

However, this is only the case if the partials have the same width and position, which is unlikely to be the case for real systems where it is expected that the average bond lengths may vary. The change that may occur on ordering, 
if the partials PDF peaks have different peak maxima, can be readily modelled using the SGM. As the total coordination number around the A and B atoms respectively must be constant, the reduction in the AA partial must result in an increase in the $\mathrm{AB}$ partial, and can be calculated for a given structure with a known number of nearest neighbours.

The amount of order in the system can be described using the WarrenCowley parameters [15, 16. These are defined as:

$$
\alpha_{l m n}^{\mathrm{AB}}=1-\frac{P_{l m n}^{\mathrm{AB}}}{c_{\mathrm{B}}}=1-\frac{P_{l m n}^{\mathrm{BA}}}{c_{\mathrm{A}}}
$$

where $P_{l m n}^{\mathrm{AB}}$ is the probability of finding a $\mathrm{B}$ atom at position $r_{l m} \vec{n}=l \overrightarrow{a_{1}}+$ $m \overrightarrow{a_{2}}+n \overrightarrow{a_{3}}$ (where $\overrightarrow{a_{1}}, \overrightarrow{a_{2}}$ and $\overrightarrow{a_{3}}$ are lattice vectors, and $l, m$ and $n$ are fractional coordinates) from an origin placed on an $\mathrm{A}$ atom, and $c_{\mathrm{A}}$ and $c_{\mathrm{B}}$ are the atomic fractions of $\mathrm{A}$ and $\mathrm{B}$ respectively in the alloy. If the atoms are randomly distributed then $\alpha_{l m n}^{\mathrm{AB}}=0$. If there is a preference for like atom interactions then $\alpha>0$, tending to a maximum of $\alpha=1$; whilst for unlike interactions $\alpha<0$, tending to a maximum of $\alpha=\frac{\left(c_{\mathrm{A}}-1\right)}{c_{\mathrm{A}}}$ when measured around $\mathrm{B}$ and $\alpha=\frac{\left(c_{\mathrm{B}}-1\right)}{c_{\mathrm{B}}}$ when measured around A.

Fig. 6 shows the change in the FWHM, peak maximum and local lattice strain on ordering in a simulated equiatomic binary alloy system, plotted against the Warren-Cowley parameter. The ordering simulated is that in which AA and $\mathrm{BB}$ partials are reduced in favour of forming $\mathrm{AB}$ partials (the type of ordering that results in $\mathrm{L}_{0}$ formation in $\mathrm{AB}$ alloys). For the $\mathrm{SM}$, a series of boxes with varying $\alpha$-parameter were built using a Monte-Carlo simulator.

From Fig. 6(a) and (d) it can be seen that as the local order increases away from random (decreasing $\alpha$ ) there is a reduction in the observed peak width. This is because more strained AA and BB partials are removed as more favourable intermediate $\mathrm{AB}$ partials form in the system, thereby reducing local strain in the system, as seen in Fig. 6(c) and (f). Comparing the models, it can be seen that with the relaxation allowed by the SM the overall magnitude of the strain is reduced compared with the SG model, although the magnitude of the change in strain upon ordering is similar for the two cases. Additionally, it can 

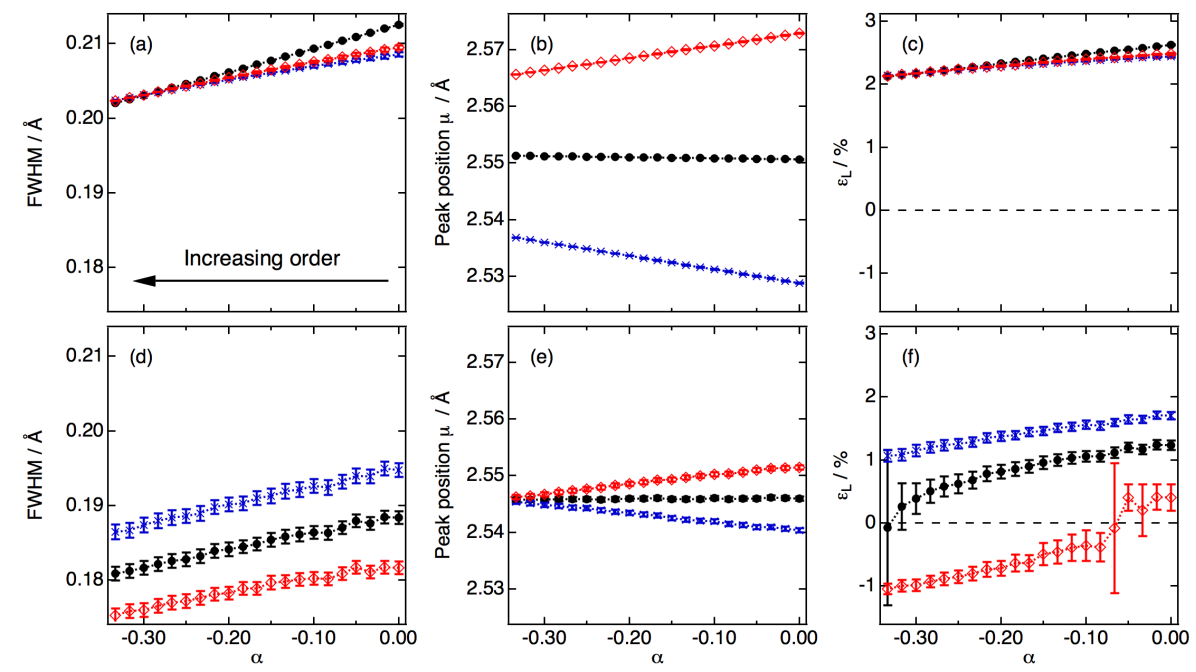

Figure 6: Variation in peak parameters for the first peak in the PDF for an AB alloy under ordering using the two models, (a) and (b) predicted using the SGM, (c) and (d) using the SM, fitting the resultant peak with a Gaussian. Horizontal axis shows Warren-Cowley parameters, with $\alpha=0$ indicating random and $\alpha<0$ an increased preference to form unlike bonds. (a) and (c) show the calculated full width at half-maximum, (b) and (d) the peak position or $\mu$ parameter. Black line (circles) indicates $b_{\mathrm{A}}=b_{\mathrm{B}}$, red line (diamonds) $2 b_{\mathrm{A}}=b_{\mathrm{B}}$ and blue line (crosses) $b_{\mathrm{A}}=2 b_{\mathrm{B}}$. Colour version available online

be seen that the magnitude of the relative scattering lengths has an important effect on the overall change in the peak width (causing an associated error in the measurement of the strain of up to $\sim 30 \%$ in this example). For the SGM, by varying the relative sizes of the symmetric $\mathrm{AA}$ and $\mathrm{BB}$ partials (the outer partials), so the width will be reduced. For the SM, the asymmetric nature of the Morse potentials and the associated peaks, results in an increase in the observed FWHM for the case where $b_{\mathrm{A}}=2 b_{\mathrm{B}}$ (blue line) case, and a decrease when $2 b_{\mathrm{A}}=b_{\mathrm{B}}$ (red line) when compared with the $b_{\mathrm{A}}=b_{\mathrm{B}}$ case.

Fig. 6(b) and (e) demonstrates that the position of the peak maximum, $\mu$, and hence the calculated lattice parameter, is also strongly affected by the relative scattering lengths, under ordering conditions. Whilst for the equal scattering length case there is little variation in the position of the peak max- 
imum, changing the relative scattering lengths changes not only the measured value of the peak maximum position, but also the rate of variation (increasing/decreasing) under conditions of ordering. Specifically, as the scattering length changes, the largest contributing partial will vary, and so therefore will the position of the peak maximum. The observed peak maximum will move away from the average value towards the radius of the most scattering species. However, this effect will be reduced by local ordering in the system, which will decrease the discrepancy by removing the outlying partials.

It should be noted that this is the result if the system orders in such a way as to favour A-B partials (and tend towards the $\mathrm{L} 1_{0}$ structure) and similar predictions could be made for other types of ordering. Importantly, the exact effect on the PDF will be dependent on the type of ordering that is occurring in the system [9], the relative scattering lengths and atomic radii of the species. As such, it is impossible to generalise this effect for all short-range order.

\section{Practical considerations}

Thus far the consideration of variation in PDF peak width has been limited to real static displacement effects; specifically variations in concentration and atomic radius, and the additional complications caused by scattering length, ordering in the system, correlated motion and dynamic displacements. In addition to these intrinsic effects, which are all the result of true local variations, there may be variations in the observed peak widths caused by external effects or non-local sample conditions (e.g. crystallite size, texture). These will need to be accounted for if the peak width is to be related to the local lattice strains in the system.

\subsection{Accounting for the difference in atomic scattering power}

As noted from the predicted distributions, the different scattering powers

of the atoms will affect the measured strain result. However, methods can be proposed to account for this. 
Firstly, choice of incident radiation. Depending on the samples under consideration the choice of X-ray or neutrons may be important. The discrepancy between true strain and observed peak width will be minimised if the different atoms have similar scattering lengths or form factors. The choice of radiation can therefore help to minimise the difference in the scattering lengths and hence reduce this effect.

Secondly, an assumption may be made that the partial distributions are the same. In the SGM, the partials are treated as independent with constant widths but different average bond lengths. In reality, each of the distributions will relax and broaden, as is observed in the case of the SM. In the extreme case, the distributions may broaden sufficiently that they become the same i.e. all the partials for the first peak in the PDF may be written as:

$$
g_{i j}(r)=N \exp \left[-\frac{(r-\mu)^{2}}{2 \sigma^{2}}\right]
$$

Note that $N, \mu$ and $\sigma$ are independent of $i, j$ in this case. Therefore, from Eq. 2 the first peak in the PDF is given as:

$$
\begin{aligned}
G(r) & =\sum_{i, j} c_{i} c_{j} \overline{b_{i}} \overline{b_{j}}\left(N \exp \left[-\frac{(r-\mu)^{2}}{2 \sigma^{2}}\right]-1\right) \\
& =\left(N \exp \left[-\frac{(r-\mu)^{2}}{2 \sigma^{2}}\right]-1\right) A_{\mathrm{FZ}}
\end{aligned}
$$

where:

$$
A_{\mathrm{FZ}}=\sum_{i, j} c_{i} c_{j} \overline{b_{i}} \overline{b_{j}}
$$

Changing the scattering length of the atomic species will change the $A_{\mathrm{FZ}}$ parameter, but not the measured peak width, $\sigma$. Therefore, if the distributions of the partials are sufficiently similar to be effectively considered to be the same, then the PDF peak width can be directly related to the local strain exhibited in the lattice. This assumption is more likely to be valid in multicomponent systems, such as equiatomic high entropy alloys, as each individual partial will have a smaller contribution to the overall PDF peak. Additionally, the range of atomic radii and complex local environments will increase the number of intermediate partials and lead to the partials converging to a similar distribution 
upon relaxation. It should be noted that this assumption will be implicit if a grey atom method is employed. This involves modelling all atoms in the system with a single atom-type, which has a scattering length equal to the average scattering length of the constituent atoms of the system (see [9]). This 'grey-atom' consideration is common, particularly when using small box modelling methods for the analysis of total scattering functions.

Alternatively, it is possible to correct for the discrepancy caused by the difference in scattering lengths by using large box modelling. In this approach, fitting the experimental PDF using the RMC algorithm [17] will yield a large box model containing tens of thousands of atoms, the predicted PDF of which provides the best fit to the available data, subject to the input constraints. From this large box model, the algorithm calculates the individual partial pair distribution functions and combines these to produce the overall PDF. Consequently, a model generated using the RMC algorithm is capable of yielding the partial pair distribution functions of a system, which are not directly experimentally accessible, but can provide invaluable insight into local effects occurring in the system.

If the partials can be accessed it is possible to decouple the effect of the scattering length on the observed peak width and more accurately assess the magnitude of the offsite displacements. As already noted, the partials are combined as in Eq. 2 to yield the $G(r)$, which will naturally be dependent on the relative scattering factors of the two materials. However, if instead, the calculation is carried out using $\overline{b_{\mathrm{A}}}=\overline{b_{\mathrm{B}}}=\cdots=1$, a function $G_{\text {strain }}(r)$ may be calculated, i.e.:

$$
G_{\text {strain }}(r)=\sum_{i, j} c_{i} c_{j}\left(g_{i j}(r)-1\right)
$$

This function will have no dependence on the relative scattering lengths and so peak widths in this 'strain PDF' will be a direct measure of the local lattice strains of a material. Fig. 7 a shows the partials for an AB equiatomic binary alloy where the A and B atoms have different scattering lengths. Fig. 7b shows how the partials vary if the scattering length changes such that $b_{\mathrm{A}}=2 b_{\mathrm{B}}$. Fig. 
77. shows the resultant summed peak that arises from the two cases. The black line represents the $G_{\text {strain }}(r)$ and the red line the as calculated $G(r)$. As can be seen, the red peak is narrower and has a lower peak maximum (as suggested from Fig. $2 \mathrm{a}$ and Fig. 2b). By calculating the $G_{\text {strain }}(r)$, this dependence on scattering length is removed. Critically, it should be noted that this method is highly reliant on an accurate determination of the partial PDFs, and so requires large box modelling (RMC fitting) to obtain this result.
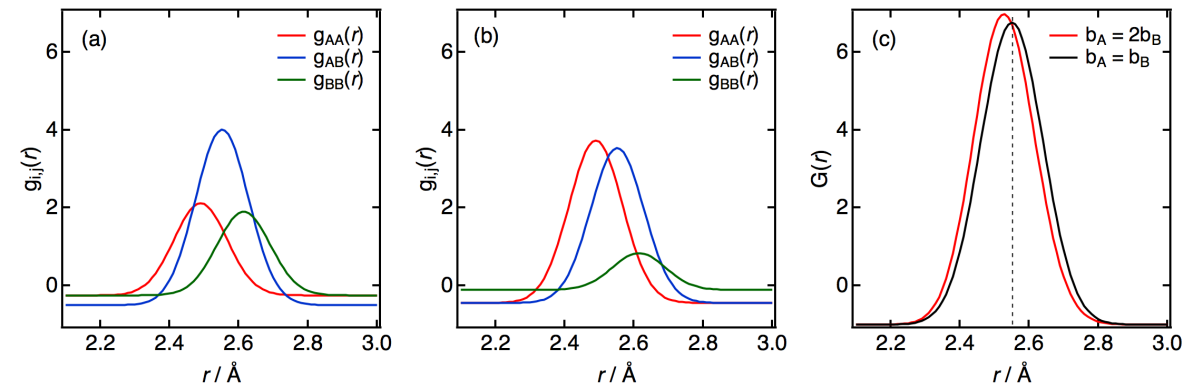

Figure 7: Change in the first peak in the PDF under variation of scattering length for an equiatomic binary alloy, $\mathrm{AB}$, with $r_{\mathrm{A}}=1.245 \AA$ and $r_{\mathrm{b}}=1.30725 \AA$, calculated using the SGM. (a) The partial pair distribution functions calculated with $b_{\mathrm{A}}=b_{\mathrm{B}}$; b) the partial pair distribution functions calculated with $b_{\mathrm{A}}=2 b_{\mathrm{B}}$; (c) the resultant peak in the PDF for the two cases shown in (a) and (b). The dotted line in (c) shows the expected peak maximum from a hard sphere model. Colour version available online.

\subsection{Calculation parameters: $Q_{\max }, Q_{\min }$ and scaling factor}

From Eq. 3 it can be seen that an integration needs to carried out over all reciprocal, $Q$, space to calculate the PDF from the total scattering function $F(Q)$.

In practice, of course, there will be a finite $Q$-range over which the Fourier transform may be carried out. Oscillations in the $F(Q)$ with a longer period will Fourier transform to components with a short-period and high-frequency in real space. Ideally, the broadest possible $Q$-range is required to provide these highfrequency terms and give the peaks maximum resolution of the peaks. Achieving a sufficiently large $Q$-range necessitates the use of either synchrotron X-ray 
radiation or spallation neutron sources, and instruments at these facilities that are optimised for total scattering data collection. However, using the largest measurable $Q$ range is not always appropriate as Fourier transforming large regions of featureless noise will result in spurious oscillations in the PDF. As such, accurate measurement of PDF peak widths requires the $Q$-range (i.e. both $Q_{\max }$ and $\left.Q_{\min }\right)$ to be chosen carefully.

Ideally, the PDF is scaled according to the number density of the material, calculated from the mass, dimensions, packing fraction and lattice parameter of the sample. As such, the correctly normalised PDF should be entirely quantitative. However, practical errors in the measurement of these normalising factors (particularly due to variations in the composition and density) often necessitate the incorporation of a scaling factor in order to be fitted satisfactorily or compared with models (e.g. PDFGui or RMC fitting).

Scalar multiples of functions are invariant under a Fourier transform. Consequently, the scaling factor applied to the $F(Q)$ could equally be applied as a scaling to the resultant PDF. Assuming that the PDF is effectively a sum of Gaussians, applying a scaling factor will simply change the scale factor of each Gaussian and not the width.

Whilst this is true mathematically; in practice this may not the case. Fitting a PDF with a sum of Gaussians uses the expression:

$$
G_{\text {calc }}(r)=y_{0}+\sum_{n} N_{n} \exp \left(-\frac{\left(x-\mu_{n}\right)^{2}}{2 \sigma_{n}^{2}}\right)
$$

where $N, \mu$ and $\sigma$ are constants defining the Gaussian function for each of the $n$ peaks, and $y_{0}$ a constant. For a correctly scaled PDF, $y_{0}=\sum_{i, j} b_{i} b_{j} c_{i} c_{j}$, i.e. the sum of the Faber-Ziman coefficients. In fitting the peak, the $y_{0}$ parameter can either be held at the desired value or allowed to refine. Depending on the range over which the refinement is carried out, it may be necessary to fix this parameter to the expected value. However, if the baseline value is fixed, then the measured height of the peak under different scalings will be different, and hence the measured FWHM (full width at half-maximum) will vary. Consequently, the scaling will affect the measured peak widths due to the 
nature of the mathematical fitting procedures carried out.

Placing theoretical measures on the magnitudes of these effects is difficult, and they should be quantified on a sample by sample basis, varying the parameters and comparing the resultant effect on the calculated peak widths.

\subsection{Instrumentation - instrument dependant broadening}

The reciprocal space resolution of a total scattering dataset will vary according to instrument and radiation choice, and may also change across the $Q$-range of the function. In the small box modelling program PDFGui, two components are used to account for instrumental resolution effects; $Q_{\text {damp }}$ and $Q_{\text {broad }}$. The $Q_{\text {damp }}$ term accounts for a $Q$-independent resolution of peaks in the $F(Q)$ and governs the observed decay envelope in the PDF data given by the shape function:

$$
S(r)=\exp \left(\frac{-\left(Q_{\mathrm{damp}}\right) r^{2}}{2}\right)
$$

where $r$ is the interatomic distance and the $Q_{\text {damp }}$ term can be estimated from the observed full width at half maximum of peaks in the $F(Q)$. The $Q_{\text {broad }}$ term is included to account for a $Q$ dependent resolution factor and is implemented through the equation:

$$
\sigma=\sigma^{\prime} \sqrt{1-\frac{\delta_{1}}{r}-\frac{\delta_{2}}{r^{2}}+\left(Q_{\text {broad }} r\right)^{2}}
$$

where $\sigma$ is the Gaussian width of a peak in the PDF, $\sigma^{\prime}$ is the width due to thermal displacements, $\delta_{1}$ and $\delta_{2}$ are parameters included to account for correlated motion in the structure, and $r$ the interatomic distance. Note that Eq. 13 is a simplified version of this equation with $\delta_{2}=0$ and $Q_{\text {broad }}=0$.

Importantly, both of these broadening terms contain an $r^{2}$ dependance, and therefore the effect on the first few PDF peaks is likely to be minimal and can largely be neglected. However, in practice it is found that their inclusion in a small box refinement has a large effect on the refined scaling parameter. This, in turn, will affect the recorded peak width (see Section 4.2) and so the use of these parameters should be carefully considered. 


\subsection{Crystallite size and strain broadening}

The Bragg peaks are, of course, an important contributor to the overall recorded PDF in a crystalline material. Unlike conventional microstrains, local lattice strains should not result in an observed broadening of the Bragg peaks, because they do not affect the interplanar spacing of the structure. However, microstrain and crystallite size, both of which show well documented broadening of the Bragg peaks, are likely to affect the static atomic displacements in a structure and so will also need to be considered.

Scherrer [18] demonstrated that the peak broadening arising from crystalline size has a $\sec \theta$ dependance (where $\theta$ is the scattering angle), whilst microstrain broadening has a $\tan \theta$ dependance. These can be reformed to similar expressions in terms of $Q$. Importantly, both lead to a broadening of the Bragg peaks with increasing $Q$. If the Bragg peaks are assumed to be Voigt functions 1 , then it can be shown that the Fourier transform of a family of $n$ Bragg peaks is given as:

$\mathscr{F}\left[f\left(N_{n}, \mu_{n}, \sigma_{n}, \Gamma_{n}, Q\right)\right]=\sum_{n} N_{n} \sqrt{2 \pi \sigma_{n}^{2}} \exp \left(-2 \pi^{2} \sigma_{n}^{2} r^{2}-\Gamma_{n} \pi|r|\right) \exp \left(-2 \pi i \mu_{n} r\right)$

where $N_{n}, \mu_{n}$ and $\sigma_{n}$ are the Gaussian components and $\Gamma_{n}$ the Lorentzian component of the Voigt function representing peak $n$.

The first exponential in this expression is a decay function, and is dependant on the width parameters of the Voigt function. The second exponential determines the period of oscillation of the contribution wave and is a function of the peak position, $\mu$, of the Bragg peak in reciprocal space. For samples with large microstrain and small crystallite size, the broadening of the Bragg peaks increases at high $Q$. Consequently, the high frequency contribution to the Fourier transform (i.e. from peaks at high $Q$ ) will be more heavily damped (as the Bragg peak width is greater, and so too the decay envelope). It is the

\footnotetext{
${ }^{1}$ Paradoxically the mathematics is simpler if a Voigt function is considered as opposed to a Pseudo-Voigt, more commonly used in diffraction fitting procedures
} 
inclusion of these high frequency oscillations that increases the resolution of the peaks in the PDF. Therefore, an increased damping of these high-frequency oscillations at high- $r$ will mean that the resolution of the PDF will decrease as $r$ increases.

In addition to the effect on the Bragg data, local strain distortion fields will form both at the surface of a particle and around the cores of dislocations present in the material. This will affect the diffuse scattering and, whilst the effect is difficult to describe in reciprocal space, it will result in a broadening of the bond distributions in low- $r$ in real space. As a result of these effects, particle size and strain broadening should also be accounted for when performing quantitative assessment of local lattice strain.

Overall, the best solution is to mitigate against these effects through careful sample preparation. Atomisation techniques typically yield particle sizes $\sim 50 \mu \mathrm{m}$ and, as such, the volume of the strained surface (assuming a thickness of around $10 \AA$ ) accounts for only $0.006 \%$ of the volume, and so strain effects can be neglected. Likewise, heat treating of the sample will allow the relaxation of the structure and reduction of the dislocation density. Typically dislocation densities in annealed nickel are of the order $10^{10}$ to $10^{12} \mathrm{~m}^{-2}$ [19, 20], which sug-

gests a strained volume of $0.0001 \%$ (assuming a strained region of $10 \AA$ around a dislocation core).

\subsection{Compositional variance}

The case examples considered in this paper were chemically homogenous systems. However, this cannot always be assumed fro real samples. As such, when experimentally applying the total scattering method for assessing local lattice strains, the chemical homogeneity of the system on the crystallite scale should first be confirmed, e.g. by SEM EDX. The identification of short-range order should then be done by considering the change in the area of PDF peaks in the system and discrepancies when fitting the system with a grey-atom model. If it is believed that short-range order is present in the system, then the widths of the peaks should be treated with caution as it is likely that the relationship 
between peak width and local strain is complicated by the presence of shortrange order.

\subsection{Texture and preferred orientation}

The total scattering technique is a powder technique and, as such, relies on the assumption that the samples constitute a crystallographic powder, i.e. one in which the crystallites occupy all possible orientations in space. However, often real samples may exhibit a preferred orientation, or crystallographic texture. Whilst the effect of texture on the Bragg pattern is well documented, the effect on the PDF is not well understood.

Crystallographic texture is identifiable from the Bragg pattern by the reduction or increase in intensity of families of Bragg peaks. This will have a corresponding affect on the PDF as it will reduce the oscillations in real space that are a function of the peak position in reciprocal space. However, unlike the effects of particle size or dislocation density there will be no systematic variation with $Q$, and so the effect on the PDF is difficult to discern.

Consequently, if a sample exhibits crystallographic texture it is currently assumed to be unsuitable for assessment by this methodology. Therefore judicious samples preparation should be carried out to ensure that the samples are not textured (e.g. atomisation, with subsequent heat-treatments).

\section{Conclusions and methodology}

This paper has considered in detail the feasibility of using total scattering methods, particularly the analysis of PDFs, for the assessment of local strain in alloys. Using two theoretical models, one considering non-interacting bond distributions and the second allowing relaxation to occur, the changes that might be expected to occur in the position of the peak maximum, the PDF peak width and hence magnitude in local lattice strain, as a result of various physical changes has been explored. It was noted that varying the concentration

of a substituting atom increased the local strain and PDF peak width, to a 
maximum at 50at.\% substitution, with an accompanying increase in the average bond length. Similarly, increasing the size of the substituting atom resulted in an increase in the observed PDF peak widths, local strain and peak maximum. In all of the examples given, the magnitude of the changes observed were highly dependant on both the difference in the radii and the relative concentrations of the atomic species. Further, it was shown how the PDF peak width is not constant across the observed $r$-range due to correlated motion effects which must be accounted for if multiple coordination shells are to be modelled. Finally, the reduction in local strain caused by ordering, with accompanying reduction in peak-width and changes in lattice parameter, was discussed.

The successful acquisition of total scattering data suitable for quantification of the local lattice strains therefore requires carefully control of the complicating factors (microstrain, crystallite size, texture, instrumentation etc.). It was shown how the measurement of PDF peak widths and positions will also be dependent on the relative scattering lengths or magnitudes of the form factors, and can result in large errors of measurement in local lattice strain (up to $\sim 40 \%$ in some of the cases considered). However, this effect may be minimised by judicious choice of radiation, average distribution ('grey-atom') assumptions or the calculation of 'strain-PDFs'.

Taken as a whole, these simulations demonstrated that analysis of the PDF

peaks in this way can yield valuable insight into the magnitude of local strains in the system and may be uniquely capable of examining the short-range effects.

\section{Methodology}

As a result of the insights gained from the theoretical case studies considered, the following methodology is suggested for the analysis of local lattice strains in alloys

- Sample preparation - Samples should be prepared in such a way as to minimise crystallographic texture, microstrain broadening and crystallite size broadening. The suggested preparation method is via either water 
or gas atomisation, with subsequent heat treatments to remove microsegregation and relax the structure to remove microstrain effects (Sections $4.4 \& 4.6$.

- Data collection - Total scattering quality data should be obtained across as broad a $Q$-range as possible. The use of high-energy synchrotron Xrays or spallation neutrons is suggested (Section 4.3). The choice of Xrays or neutrons should be determined by the relative scattering lengths and contributing form factor effects that may be observed. The direct determination of local strains is made simpler if the assumption can be made that the different elements have similar scattering lengths (Section 4.1. It should be noted that this is actually the opposite advice normally given for the planning of a total scattering experiment! All data, for a comparative study, should be collected on the same instrument, to limit instrumental $Q$-resolution effects.

- Data processing - A number of internal checks need to be carried out during the data processing. The following steps are recommended:

1. SEM imaging and EDX analysis - to determine the composition and chemical homogeneity of the material, and the crystallite (particle) size to ensure that these factors do not affect the PDF data.

2. Powder pattern refinement - Rietveld refinement should be carried out to determine accurate values of the lattice parameter and to ensure that the sample contains no texture. If the sample is textured, subsequent analyses will not be accurate using existing data processing tools.

3. Total scattering data processing and Fourier transform - ensure that $Q_{\min }$ and $Q_{\max }$ are constant for all samples (Section 4.2), and that the $Q$-range is chosen from a consideration of how the peak widths change under variations in these parameters.

- Modelling - The following modelling steps are suggested for the extraction 
of quantitative local lattice strain data:

1. Grey atom modelling - A grey atom model, either large or small box, can be useful in suggesting whether there is any change in chemical order (short-range order) in the system from a change in area of the PDF peaks. If so, it may be that assessments of the local lattice strain are no longer feasible.

2. Calculation of a 'strain PDF' - Fitting of the PDF can be carried out using a multiple component large box model. From the output structure the atoms can be given the same scattering length and the PDF recalculated to yield a 'strain PDF' from which the peak widths will be directly related to the observed strain (Section 4.1).

3. Peak width determination - The observed peak widths can be measured using either the FWHM or Gaussian fitting method. Errors should be calculated based on the maximum error inherent in the system (e.g. from error in varying one of the fitting parameters such as the scale factor). If either a 'grey-atom' assumption or the 'strainPDF' method are used this should be explicitly stated.

It is hoped that this methodology will facilitate the accurate determination and comparison of local lattice strains in different alloy systems. This could help to provide valuable insight and clarity in many fields, such as the study of the High-Entropy Alloys, where the nature of these subtle effects have been used to explain observed physical properties.

\section{Acknowledgements}

This work was supported by the STFC ISIS Facility and the Rolls-Royce /EPSRC Strategic Partnership under EP/H022309/1 and EP/ M005607/1. L.

R. Owen would like to thank Gonville and Caius College for their support.

[1] J.-W. Yeh, S.-K. Chen, S.-J. Lin, J.-Y. Gan, T.-S. Chin, T.-T. Shun, C.-H. Tsau, S.-Y. Chang, Nanostructured high-entropy alloys with multiple prin- 
cipal elements: Novel alloy design concepts and outcomes, Advanced Engineering Materials 6 (5) (2004) 299-303. doi:10.1002/adem.200300567. URL http://dx.doi.org/10.1002/adem.200300567

[2] J. Yeh, Recent progress in high-entropy alloys, Annales de chimie - Science des Materiaux 31 (2006) 633-648.

[3] J. Yeh, Physical metallurgy of high-entropy alloys, Journal of materials 67 (2015) 2254-2261.

[4] L. Owen, E. Pickering, H. Playford, H. Stone, M. Tucker, N. Jones, An assessment of the lattice strain in the CrMnFeCoNi high-entropy alloy, Acta Materialia 122 (2017) 11-18.

[5] B. Cantor, I. Change, P. Knight, A. Vincent, Microstructural development in equiatomic multicomponent alloys, Material Science and Engineering A 213 (2004) 375-377.

[6] H. Y. Playford, L. R. Owen, I. Levin, M. G. Tucker, New insights into complex materials using reverse monte carlo modeling, Annual Review of Materials Research 44 (2014) 429-449.

[7] P. Debye, Interferenz von Röntgenstahlen und wärmebewegung, Annalen der Physik 348 (1) (1913) 49-92.

[8] I. Waller, Zur frage der einwirkung der wärmebewegung auf die intergerenz von röntgenstrahlen, Zeitschrift fur Physik 17 (1923) 398-408.

[9] L. R. Owen, H. Y. Playford, H. J. Stone, M. G. Tucker, A new approach to the analysis of short-range order in alloys using total scattering, Acta Materialia 115 (2016) 155-166.

[10] L. Owen, H. Playford, H. Stone, M. Tucker, Analysis of short-range order in $\mathrm{Cu}_{3} \mathrm{Au}$ using X-ray pair distribution, Acta Materialia 125 (2017) 15-26.

[11] L. Vegard, Die konstitution der mischkristalle und die raumfullung der atome, Zeitschrift fur Physik 5 (1) (1921) 17-26. 
[12] W. Hume-Rothery, G. V. Raynor, The Structure of Metals and Alloys, Institute of Metals, 1954.

[13] C. L. Farrow, P. Juhas, J. W. Lui, D. Bryndin, E. S. Bozin, J. Bloch, T. Proffen, S. Billinge, PDFfit2 and PDFGui: computer programs for studying nanostructure in crystals., Journal of Physics: Condensed Matter $19(33)$.

[14] T. Proffen, V. Petkov, S. Billinge, T. Vogt, Chemical short range order obtained from the atomic pair distribution function, Zeitschrift fur Kristallographie 216 (2) (2001) 47-50.

[15] J. M. Cowley, An approximate theory of order in alloys, Physical Review 77 (5) (1950) 669-675.

[16] J. M. Cowley, Short-range order and long-range order parameters., Physical Review 138 (5A) (1965) 1384-1389.

[17] R. L. McGreevy, L. Pusztai, Reverse Monte Carlo simulation: A new technique for the determination of disordered structures, Molecular Simulation 1 (6) (1988) 359-367.

[18] P. Scherrer, Bestimmung der grosse und der inneren struktur von kolloidteilchen mittels rontgenstrahlen, Gottinger Nachrichten 2 (1918) 98100.

[19] L. Clareborough, M. Hargreaces, M. Loretto, G. West, The influence of impurities on the annealing of nickel after cold work, Acta Metallurgica $8(797-803)$.

[20] P. Gay, P. Hirsch, A. Kelly, The estimation of dislocation densities in metals from X-ray data, Acta Metallurgica 1 (1953) 315-319. 